Research Article

\title{
Traditional Turkish Coffee with Medicinal Effect
}

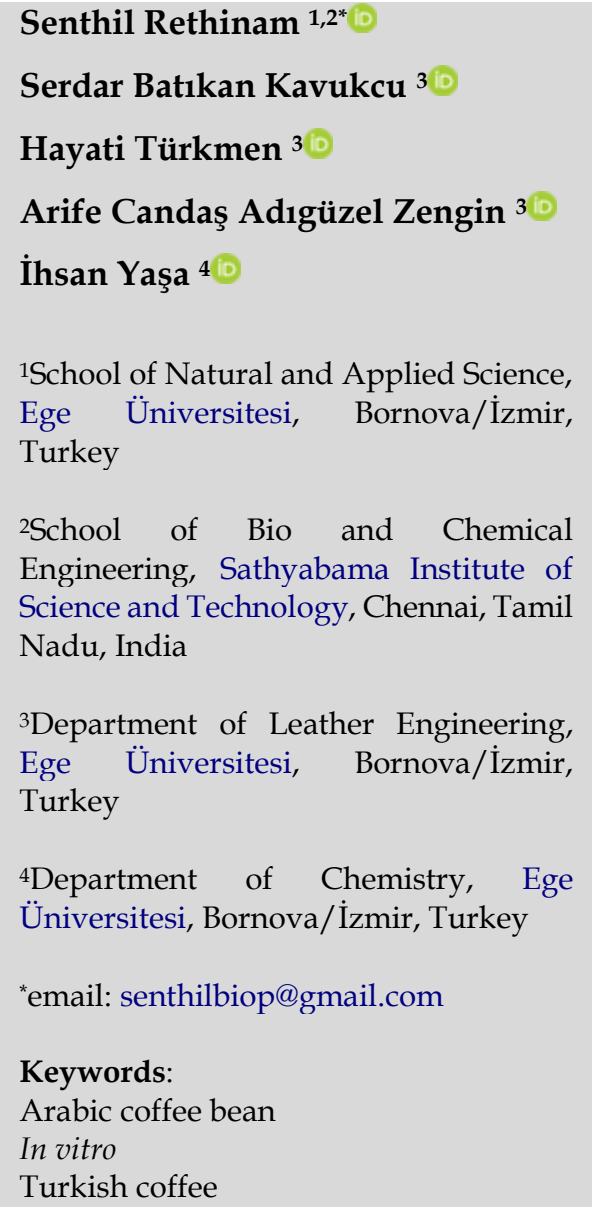

\begin{abstract}
Traditional Turkish coffee (TTC) is highly associated with caffeine and is known as a mind and heart stimulant as it helps keep tiredness at bay. Daily consumption of TTC naturally benefits human health such as anti-cancer, anti-diabetic, improved energy, anti-depression, reduced risk of heart disease, etc. The TTC was derived from particular types of Arabic coffee beans (ACB), and the preparation method of TTC is unique from other types of coffee. The study's main objective was to investigate the therapeutic and biological effects of TTC. The ACB powder was characterized physicochemically using UV-Vis spectroscopy, Fourier transforms infrared spectroscopy (FTIR), scanning electron microscopy (SEM), and energy-dispersive X-ray spectroscopy (EDX). In vitro analysis using HaCaT (Human keratinocyte cell line) proved the biocompatibility of ACB powder. Case studies focusing on healthy individuals as the research populace were conducted using TTC. Consumption of TTC was found beneficially compared to other types of coffee. The TTC was obtained from $A C B$, which was characterized by spectroscopic techniques and displayed biocompatibility due to the results on HaCaT cell lines. The TTC has beneficial therapeutic effects on individuals. According to statistical analysis, the disease-affected ratio of diabetes, heart disease, and depression was significantly decreased.
\end{abstract}

Received: June 28th 2021

Accepted: October 6th 2021

Published: November 30th, 2021

(c) 2021 Senthil Rethinam, Serdar Batıkan Kavukcu, Hayati Türkmen, Arife Candaş Adıgüzel Zengin, İhsan Yaşa. Published by Institute for Research and Community Services Universitas Muhammadiyah Palangkaraya. This is an Open Access article under the CC-BY-SA License (http://creativecommons.org/licenses/by-sa/4.0/). DOI: https:// doi.org/10.33084/bjop.v4i4.2378

\section{INTRODUCTION}

Coffee is one of the most consumed beverages worldwide and has a chief role in aiding the health benefits owing to its physiological effects on the central nervous and biological system. Coffee is known for its rich aroma, taste, and flavor ${ }^{1}$. It comprises many different chemical structures and contains various substances like phenolic and alkaloids elements, vitamins, lipids, minerals, nitrogen compounds, chlorogenic acids, and carbohydrates ${ }^{2}$. Drinking coffee is a common habit in developing and developed countries. According to the latest estimate from the International Coffee Organization (ICO), approximately 2.5 billion cups of coffee are intake in the world every day ${ }^{3}$.

Caffeine (1,3,7-trimethylpurine-2,6-dione) has known as an alkaloid that consists of two fused rings on associated purines ${ }^{4}$. Myriad people worldwide prefer coffee. Drinking coffee in constraint has several bioactive/volatile compounds, and it adds to the wellbeing of human health ${ }^{5}$. Effective use of caffeine consumption has reported better experimentation on human beings and animals. It gives ergonomics the 
ability to be physically active without a concurrent increase in the sensation of effort ${ }^{6}$.

There has been an increasing concern regarding Alzheimer's, Parkinson's, type 2 diabetes mellitus, cognitive functions, sleep quality, anxiety, and depression $^{78}$. Traditional medicine is common in developed and developing countries at the primary healthcare level. The World Health Organization (WHO) reported that people preferred traditional medicines at least $75-95 \%$ of the population in developing countries, and a majority of it involved the use of plant-based medicinal products or their active constituents, ${ }^{9,10}$.

Traditional Turkish coffee (TTC) is traditionally used in Turkey and most European countries. The preparation method of the TTC bean concentrates on fine powdering. The fine powder is then let to boil slowly in water (one spoon of coffee powder and one cup of water). The resultant half-boiled coffee solution is transferred to a cup and is left undisturbed while it settles at the bottom of the cup. Traditional Turkish coffee was found caffeine and other biologically active compounds in higher amounts when compared to other coffee preparation methods ${ }^{11}$. Traditional Turkish coffee plays a versatile role in traditional medicines like caffeine and other biological compounds for anti-cancer, anti-inflammatory, antidiabetic, antimicrobial, reducing the risk of mortality, cirrhosis, chronic and progressive diseases, coronary heart disease, Alzheimer's, and Parkinson's diseases ${ }^{1213}$. In this study, we present the preparation of TTC from Arabic coffee beans (ACB) and the effect of TTC consumption on healthy individuals. The $\mathrm{ACB}$ powder was characterized via UV-Vis spectrophotometer, Fourier transform infrared (FTIR) spectroscopy, scanning electron microscope (SEM), and energy-dispersive X-ray spectroscopy (EDX) analysis. The antimicrobial activity of ACB was evaluated against Staphylococcus aureus and Escherichia coli. The biocompatibility analysis of $\mathrm{ACB}$ was measured on human keratinocyte (HaCaT) cell line. The TTC consumption study on healthy individuals was conducted for 12 months. The medical history of individuals was evaluated.

\section{MATERIALS AND METHODS}

\section{Materials}

Traditional Turkish Coffee bean was collected from a local supermarket, Izmir/Bornova, Turkey (Company Certificate Number: TR-34-K-004732), and the taxonomic position of the ACB was identified and authenticated. The ACB was collected in large quantities, dried in the shade, and powdered. The HaCaT cell line was purchased from the National Centre for Cell Science (NCCS), Pune, India.

\section{Methods}

\section{Physicochemical characterization}

The optical transmission/absorption spectra of $\mathrm{ACB}$ powder dispersed in water were recorded using a UVVis spectrophotometer (Hitachi, U3010) from 200 to 800 $\mathrm{nm}$. Fourier transform infrared spectroscopy measurements were carried out to determine the formation and changes in the functional groups of $\mathrm{ACB}$ powder. The spectra were measured at a resolution of 4 $\mathrm{cm}^{-1}$ in the frequency range of $4000-500 \mathrm{~cm}^{-1}$ using the Nicolet 360 FTIR Spectrometer. The surface morphology of the samples was visualized by SEM and EDX analysis (SEM Model LEICA stereo scan 440).

\section{Bacterial culture and antibacterial activity}

The antimicrobial activity was quantitatively evaluated against S. aureus (ATCC 25923) and E. coli (ATCC 11303). All the cultures were sub-cultured periodically and maintained on nutrient agar (NA) medium at room temperature $\left(30 \pm 2^{\circ} \mathrm{C}\right)$ for further experiments. The antimicrobial activity effect of the prepared $\mathrm{ACB}$ powder sample was found by the modified agar well diffusion 
method. Muller-Hinton agars were sterilized and poured on Petri dish and allowed to solidify under laminar airflow. About $100 \mu \mathrm{L}\left(10^{8} \mathrm{CFU} / \mathrm{mL}\right.$ (colony forming units)) of each bacterial culture was spread on the agar surface using a sterile glass spreader. Samples of $1 \mathrm{~cm}$ diameter were spread on the agar plate. The antibacterial activity was evaluated by measuring the diameter of the zone of inhibition against the organism. The control samples were prepared using amoxicillin and azithromycin antibacterial disc.

\section{Biocompatibility analysis}

The cell line culture was maintained in Dulbecco's Modified Eagles Medium (DMEM) with 10\% fetal bovine serum (FBS) at $37^{\circ} \mathrm{C}$ with $95 \%$ air and $5 \% \mathrm{CO}_{2}$. Cell viability in terms of metabolic activity was evaluated calorimetrically by 3-(4,5-dimethylthiazol-2-yl)-2, 5diphenyltetrazolium bromide (MTT) assay. Cells (25 × $10^{3}$ cells $/ \mathrm{mL}$ ) were seeded on ACB powder in a 24 well plate. After 30 minutes, a 10\% DMEM was added, and the cells were incubated for one and two days. The cell growth on the culture plate without ACB powder was taken as control. The percentage of cell viability was calculated compared to the control. The cells were stained with acridine orange, and their morphology was viewed using fluorescent microscopy ${ }^{14}$.

\section{Preparation of Traditional Turkish Coffee}

The preparation of TTC was done according to Severini et $a^{15}$. In brief, $7 \mathrm{~g}$ of coffee powder was taken and dispersed in $100 \mathrm{~mL}$ of cold water and stirred in the presence of sugar, having a homogeneous mixture before cooking. The resultant solutions were heated in a traditional coffee pot for three minutes at $100^{\circ} \mathrm{C}$. Coffee was boiled to obtain foam, and cups in which coffee was served should also be hot.

\section{Traditional Turkish Coffee assessment in healthy adults}

Ethical approval for this research was obtained via the local ethics committee-health science subcommittee of Ege Üniversitesi (EGE BAYEC) (with the following final decision and protocol number 19.03.2020/12-2020). The healthy adults were provided clear explanations about the study purpose, after which formal informed consent was obtained. The participants were given a written survey to establish their demographic information, such as age, education level, and coffee consumption. Following primary data collection from the participants, anthropometric measurements such as body weight and height were gathered. The data for this sectional study was collected through face-to-face interviews. Participants were thrown out if they had a history of diabetic, metabolic, cardiovascular, and musculoskeletal disease or taking any other medicine, as per the medical history questionnaire.

The effectiveness of this TTC was assessed on healthy adults with different age groups. Healthy individuals consisting of twelve men and twelve women aged 18-35 years were recruited to participate in this study (age 26.0 \pm 2.3 y.o.; height $1.68 \pm 0.04 \mathrm{~m}$; body mass $74.2 \pm 12.8 \mathrm{~kg}$ ). Individuals were randomly divided into three groups depending upon the coffee used. Group I $(n=6)$ control, Group II ( $n=6)$ were individuals who used their regular instant coffee (RIC), Group III $(n=6)$ were individuals who used TTC with sugar, and Group IV $(n=6)$ were individuals who used TTC without sugar. All individuals gave informed consent for the study. The study was conducted for 12 months, and the medical history of individuals such as diabetics, coronary heart disease, and depression was also observed and evaluated. 


\section{RESULTS AND DISCUSSION}

\section{Ultraviolet-visible spectrophotometer}

The ultraviolet-visible spectrum of the ACB powder was shown in Figure 1. The strong interfering band was detected at wavelengths 210, 284, and $322 \mathrm{~nm}$. The composition of chlorogenic acid generates complexes with caffeine ${ }^{16}$. The important absorption peaks of chemical components are found in roasted coffee-the caffeine absorption peak at $281 \mathrm{~nm}$ and $320 \mathrm{~nm}$ due to the absorption of caffeic acid ${ }^{17}$.

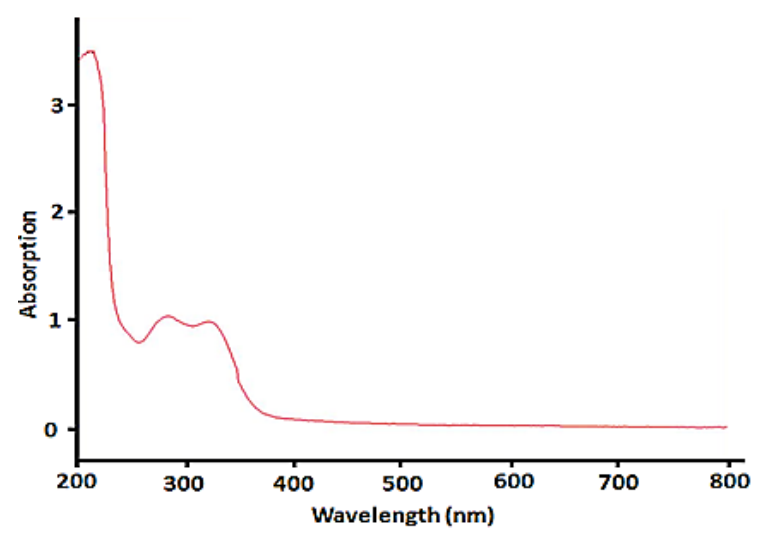

Figure 1. UV-vis spectrum of ACB powder

\section{Fourier transform infrared spectroscopy}

The FTIR analysis was used to identify the functional groups of ACB powder (Figure 2). The description for each peak as a functional group in the FTIR spectra was mentioned. The absorption band at $1160-1370 \mathrm{~cm}^{-1}$ and $1655-1745 \mathrm{~cm}^{-1}$ were characteristics of the differences in the caffeine and chlorogenic acids. Spectra of organic molecules displayed 1020-1600 $\mathrm{cm}^{-1}$, which could be a complex region of $\mathrm{P}-\mathrm{O}, \mathrm{C}-\mathrm{O}, \mathrm{C}-\mathrm{H}$, and C-N vibrations. Flament ${ }^{18}$ has reported that roasted coffee flavor compounds form containing complex mixture like acid compounds, aldehyde, ketone, and ester plays a principal role in producing the fragrance of coffee. Nebesny and Budryn ${ }^{19}$ have demonstrated that coffee has potential antioxidants such as chlorogenic acid (phenolic compounds), caffeine, and hydroxycinnamic acids.

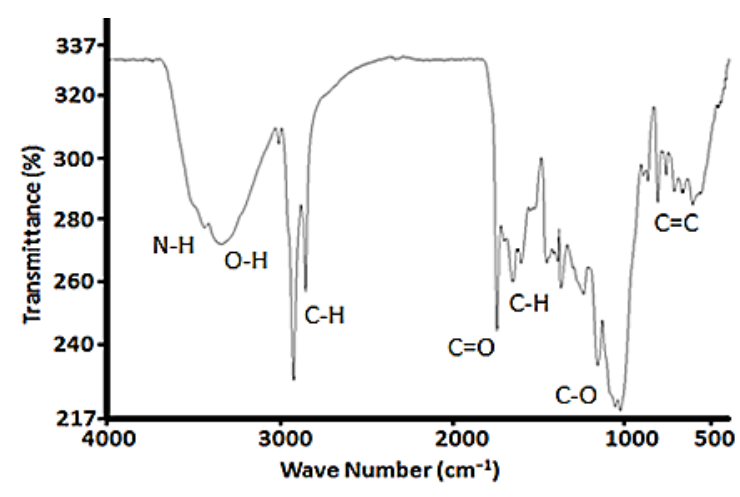

Figure 2. FTIR spectrum of ACB powder

\section{SEM and EDX analysis}

The SEM was used to observe the surface morphology of the prepared ACB powder (Figure 3). The average particle size of Turkish coffee powder was $508 \pm 7 \mathrm{~mm}$, indicating its smooth surface and spherical shape. The image observed the ACB powder surface a variety of flaky protuberances. Hao et $a^{20}$. reported that the morphology was analyzed as particle size, shape, and surface in the previous study. The EDX analysis results (Figure 4) of the ACB powder contained the minerals element such as CK, OK, MgK, KrL, PK, and AuM.

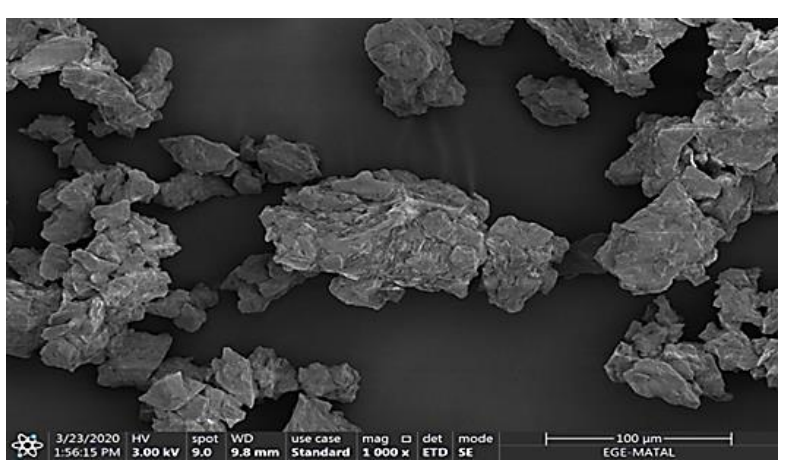

Figure 3. SEM of ACB powder

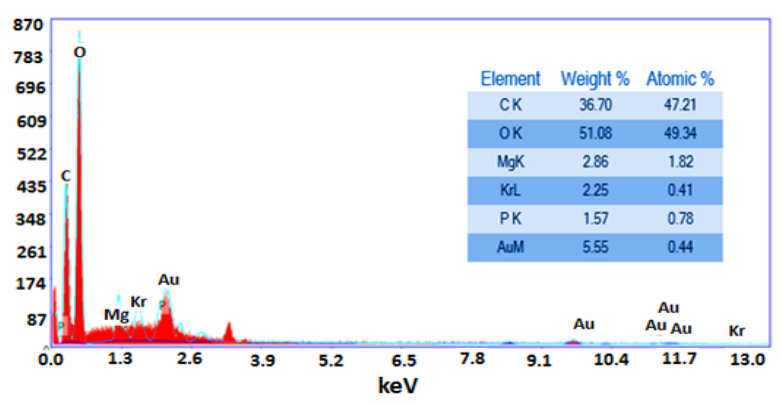

Figure 4. EDX of ACB powder 


\section{Bacterial culture and antibacterial activity}

The ACB powder results in Figure 5 depict the antibacterial activity (minimum inhibitory concentration or MIC) against S. aureus and E. coli. The ACB highest antibacterial activity (Table I), whereas control samples (amoxicillin and azithromycin) were the low zone of inhibition $^{21}$. The antimicrobial effectiveness of $\mathrm{ACB}$ powder is due to the characteristics of the potentiality of caffeine ${ }^{22}$. Nuhu ${ }^{23}$ has studied that the extraction of coffee powder of the bioactive compounds has a predominant function in antibacterial activity. Caffeine contains purine alkaloid compounds to DNA by inhibiting the incorporation of adenine and thymidine and inhibiting protein synthesis. Similar studies on S. aureus and E. coli have resulted that caffeine inhibits protein synthesis, RNA impairs, and DNA synthesis ${ }^{24}$. Caffeine is a natural antimicrobial activity, which growth of microbial strains reduction is nearly accomplice with changes in cell morphology in the various bacterial strains like S. aureus and E. coli25.

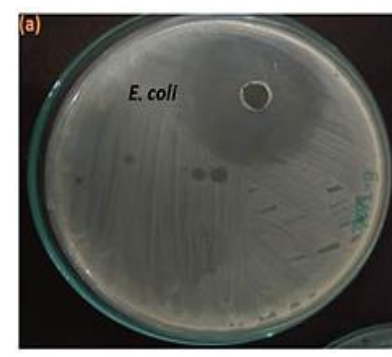

a

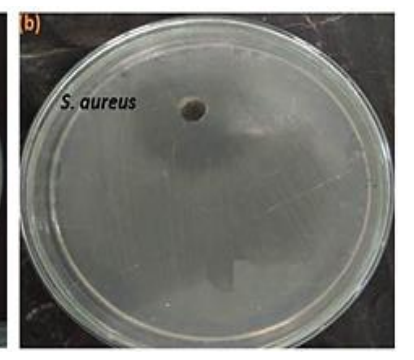

b
Figure 5. Inhibition zone of ACB powder against E. coli (a) and S. aureus (b)

Table I. Antimicrobial activity of ACB powder against E. coli and S. aureus

\begin{tabular}{lc}
\hline \multicolumn{1}{c}{ Organism } & Inhibition zone $(\mathbf{m m})$ \\
\hline E. coli & $3.96 \pm 0.02$ \\
S. aureus & $3.97 \pm 0.02$ \\
\hline
\end{tabular}

\section{Biocompatibility analysis}

Examination of the biocompatibility of HaCaT cells in the uptake of ACB powder was analyzed using an MTT assay. Figure 6 shows the results indicating the percentage of viable cells was found to be more in $\mathrm{ACB}$ powder when compared to control, which indicates the $100 \%$ biocompatibility of ACB powder to the HaCaT cells. ACB powder was evaluated through fluorescence microscopy, as shown in Figure 7. The previous study has proved the number of live cells after staining with a vital dye ${ }^{26}$. Silverberg et al..$^{27}$ reported that fibroblast treated caffeine was protected toxicity, leading to more cell viability, enhanced cell morphology, mechanism, and effective antioxidant properties. Early studies have obtained favorable results in cell viability and changes of morphologies with caffeine-treated $\mathrm{HaCaT}$ cells. Although cancer cell death by caffeine was investigated, caffeine is well recognized as a cancer cell death inducer, which helps anti-carcinogenic properties ${ }^{28}$.

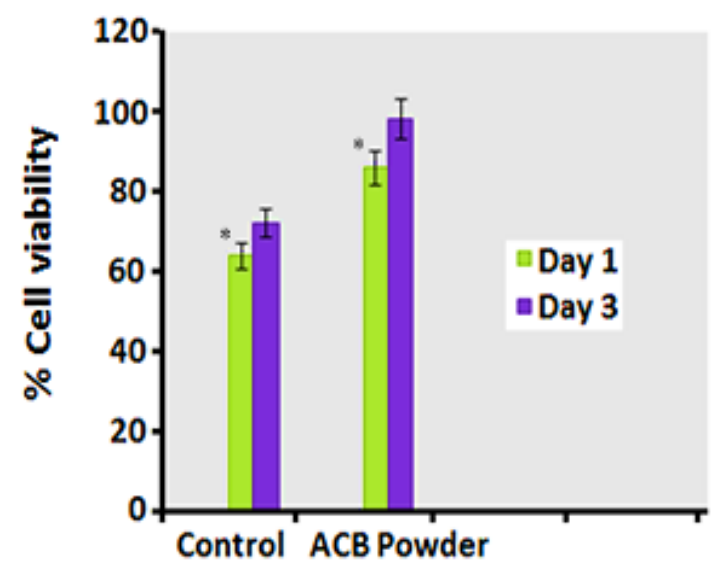

Figure 6. In vitro study of ACB powder on HaCaT cell line. The asterisks $\left({ }^{*}\right)$ indicate statistically significant differences compared to the control $\mathrm{p}<0.05$

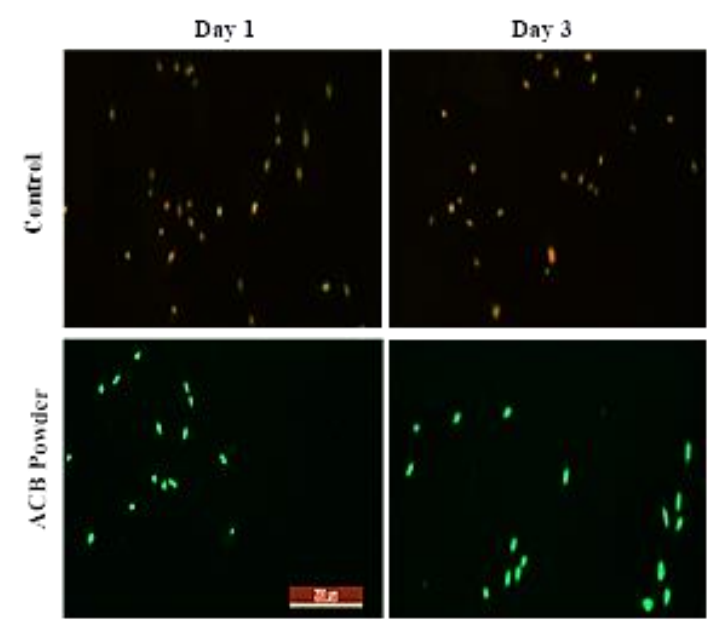

Figure 7. Florescence micrographs (20X) of HaCaT cell line cultured on day 1 and 3 


\section{Traditional Turkish Coffee assessment in healthy adults}

The constancy and habits of three types of coffee, such as standard instant coffee, TTC with sugar, and TTC without sugar, were determined throughout 12 months. Each participant gave his or her informed consent to participate in the trial after hearing about all the procedures, risks, and benefits. The aroma and flavor of TTC could effectively add to the health benefits and help significantly reduce health risks (Figure 8). The results of this study also comply with the statement, where the development of diseases like diabetes, heart disease, and depression was significantly less in group III and group IV compared to group II. The results of Morphett et al.29 contradicts the above results, wherein they report the 50 years old female who used caffeine consumption (1week) stage 1, caffeine <= $160 \mathrm{mg} /$ day; (1-week) stage 2, caffeine $<=80 \mathrm{mg} /$ day; (7-week) stage 2 , caffeine $<=35$ $\mathrm{mg} /$ day) had a significant reduction in behavioral change. It could also be stated that the antioxidant principles found in TTC might have also contributed towards oxidative stress prevention. Evaluation of coffee in the minimum number of people is an important study limitation. Nevertheless, since the study results were promising, future studies are aimed to be conducted on a large scale. Alves and Oliveira ${ }^{30}$ have reported that Turkey coffee consumption of about 1-2 cups per day may reduce the risk of colorectal cancer, diabetes, Alzheimer's disease, Parkinson's disease, and heart disease.

During the TTC trial, this study found significant increases in subjective measures of improved energy and trends in depression. It is essential to recognize the strengths and limitations of this study. The research study plays a critical role in health psychology and stored details of individuals in context. During the experimental trial, all the Groups had an imbalanced blood pressure level. Excessive coffee drinking might also contribute to high blood pressure. Many studies have reported the effects of commonly consumed coffee beverages on blood pressure ${ }^{31}$.

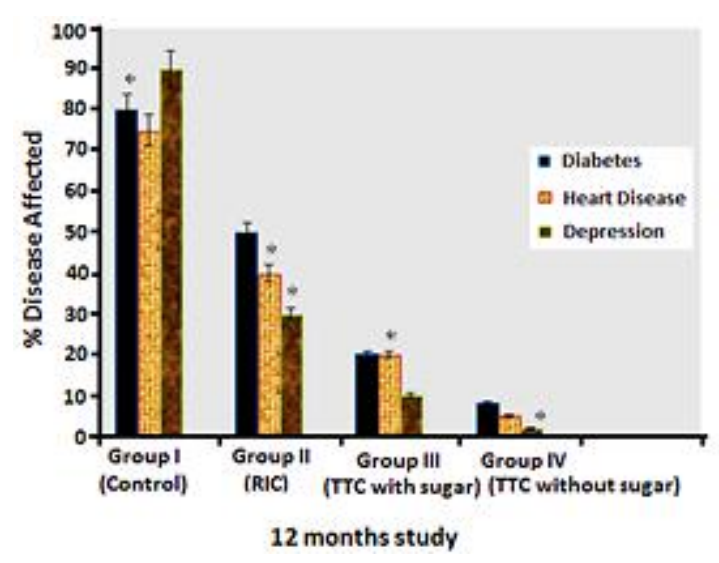

Figure 8. Comparison of health benefits of control, RIC, TTC with sugar, and TTC without sugar

\section{CONCLUSION}

The present study was carried out to analyze the properties of $\mathrm{ACB}$ powder. The TTC prepared from ACB powder and consumed regularly may reduce the risk of diabetes, heart disease, and depression. In vitro analysis using $\mathrm{HaCaT}$ cell line proved the biocompatibility and its more viable cells present in ACB powder. The research study was checked for their efficacy as therapeutic Turkish coffee for different age groups of people. On the other hand, TTC appears to have more benefits, such as reduced acidity and high antioxidant activity, as well as no stomach issues.

\section{ACKNOWLEDGMENT}

All authors acknowledge the funding support granted by TÜBITAK BIDEB 2232 - International Fellowship for Outstanding Researchers (Project No: 118C350).

\section{AUTHORS' CONTRIBUTION}

All authors contribute equally. 


\section{DATA AVAILABILITY}

The supporting data of the article are accessible from the corresponding author upon reasonable request.

\section{CONFLICT OF INTEREST}

The authors of this manuscript have no conflicts of interest related to the content of the study.

\section{REFERENCES}

1. Samoggia A, Riedel B. Consumers' Perceptions of Coffee Health Benefits and Motives for Coffee Consumption and Purchasing. Nutrients. 2019;11(3):653. doi:10.3390/nu11030653

2. Tungmunnithum D, Thongboonyou A, Pholboon A, Yangsabai A. Flavonoids and Other Phenolic Compounds from Medicinal Plants for Pharmaceutical and Medical Aspects: An Overview. Medicines. 2018;5(3):93. doi:10.3390/medicines5030093

3. Czarniecka-Skubina E, Pielak M, Sałek P, Korzeniowska-Ginter R, Owczarek T. Consumer Choices and Habits Related to Coffee Consumption by Poles. Int J Environ Res Public Health. 2021;18(8):3948. doi:10.3390/ijerph18083948

4. Ashihara $\mathrm{H}$, Sano $\mathrm{H}$, Crozier A. Caffeine and related purine alkaloids: biosynthesis, catabolism, function and genetic engineering. Phytochemistry. 2008;69(4):841-56. doi:10.1016/j.phytochem.2007.10.029

5. Bae JH, Park JH, Im SS, Song DK. Coffee and health. Integr Med Res. 2014;3(4):189-91. doi:10.1016/j.imr.2014.08.002

6. Temple JL, Bernard C, Lipshultz SE, Czachor JD, Westphal JA, Mestre MA. The Safety of Ingested Caffeine: A Comprehensive Review. Front Psychiatry. 2017;8:80. doi:10.3389/fpsyt.2017.00080

7. Livingston G, Huntley J, Sommerlad A, Ames D, Ballard C, Banerjee $S$, et al. Dementia prevention, intervention, and care: 2020 report of the Lancet Commission. Lancet. 2020;396(10248):413-46. doi:10.1016/s0140-6736(20)30367-6
8. Butt MS, Sultan MT. Coffee and its consumption: benefits and risks. Crit Rev Food Sci Nutr. 2011;51(4):363-73. doi:10.1080/10408390903586412

9. James PB, Wardle J, Steel A, Adams J. Traditional, complementary and alternative medicine use in SubSaharan Africa: a systematic review. BMJ Glob Health. 2018;3(5):e000895. doi:10.1136/bmjgh-2018000895

10. Sofowora A, Ogunbodede E, Onayade A. The Role and Place of Medicinal Plants in the Strategies for Disease Prevention. Afr J Tradit Complement Altern Med. 2013;10(5):210-29. doi:10.4314/ajtcam.v10i5.2

11. Kuçükkömürler S., Özgen V. Coffee and Turkish Coffee Culture. Pak J Nutr. 2009;8(10):1693-700. doi:10.3923/pjn.2009.1693.1700

12. Islam MT, Tabrez $S$, Jabir NR, Kamal MA, Araujo LdS, Santos JVDO, et al. An Insight into the Therapeutic Potential of Major Coffee Components. Curr Drug Metab. 2018;19(6):544-56. doi:10.2174/1389200219666180302154551

13. Oliveira M, Casal S, Morais S, Alves C, Dias F, Ramos $S$, et al. Intra- and interspecific mineral composition variability of commercial instant coffees and coffee substitutes: Contribution to mineral intake. Food Chem. 2012;130(3):702-9. doi:10.1016/j.foodchem.2011.07.113

14. Rethinam S, Basaran B, Vijayan S, Mert A, Bayraktar $\mathrm{O}$, Aruni AW. Electrospun nano-bio membrane for bone tissue engineering application- a new approach . Mater Chem Phys. 2020;249:123010. doi:10.1016/j.matchemphys.2020.123010

15. Severini C, Derossi A, Ricci I, Caporizzi R, Fiore A Roasting Conditions, Grinding Level and Brewing Method Highly Affect the Healthy Benefits of a Coffee Cup. Int J Clin Nutr Diet. 2018;4:127-32. doi:10.15344/2456-8171/2018/127

16. Nabavi SF, Tejada S, Setzer WN, Gortzi O, Sureda A, Braidy N, et al. Chlorogenic Acid and Mental Diseases: From Chemistry to Medicine. Curr Neuropharmacol. 2017;15(4):471-9. doi:10.2174/1570159X14666160325120625

17. Souto UTdCP, Barbossa MF, Dantas HV, de Pontes AS, Lyra WdS, Diniz PHGD, et al. Identification of adulteration in ground roasted coffees using UV-Vis spectroscopy and SPA-LDA. LWT Food Sci Technol. 2015;63(2):1037-41. doi:10.1016/j.lwt.2015.04.003 
18. Flament I. Coffee Flavor Chemistry. New York (US): John Wiley \& Sons; 2002.

19. Nebesny E, Budryn G. Antioxidative activity of green and roasted coffee beans as influenced by convection and microwave roasting methods and content of certain compounds. Eur Food Res Technol. 2003;217:157-63. doi:10.1007/s00217-003-0705-4

20. Hao L, Wang P, Valiyaveettil S. Successive extraction of $\mathrm{As}(\mathrm{V}), \mathrm{Cu}(\mathrm{II})$ and $\mathrm{P}(\mathrm{V})$ ions from water using spent coffee powder as renewable bioadsorbents. Sci Rep. 2017;7:42881. doi:10.1038/srep42881

21. Kibret M, Abera B. Antimicrobial susceptibility patterns of E. coli from clinical sources in northeast Ethiopia. Afr Health Sci. 2011;Suppl 1(Suppl 1):S40-5. doi:10.4314/ahs.v11i3.70069

22. Almeida AAP, Farah A, Silva DAM, Nunan EA, Glória MBA. Antibacterial activity of coffee extracts and selected coffee chemical compounds against enterobacteria. J Agric Food Chem. 2006;54(23):873843. doi:10.1021/jf0617317

23. Nuhu AA. Bioactive Micronutrients in Coffee: Recent Analytical Approaches for Characterization and Quantification. ISRN Nutr. 2014;2014:384230. doi:10.1155/2014/384230

24. Sandlie I, Solberg K, Kleppe K. The effect of caffeine on cell growth and metabolism of thymidine in Escherichia coli. Mutat Res. 1980;73(1):29-41. doi:10.1016/0027-5107(80)90133-5

25. Ackerley DF, Barak Y, Lynch SV, Curtin J, Matin A. Effect of chromate stress on Escherichia coli K-12. J Bacteriol. 2006;188(9):3371-81. doi:10.1128/jb.188.9.3371-3381.2006

26. Triglia D, Braa SS, Yonan C, Naughton GK. In vitro toxicity of various classes of test agents using the neutral red assay on a human three-dimensional physiologic skin model. In Vitro Cell Dev Biol. 1991;27A(3 Pt 1):239-44. doi:10.1007/bf02630923

27. Silverberg JI, Patel M, Brody N, Jagdeo J. Caffeine protects human skin fibroblasts from acute reactive oxygen species-induced necrosis. J Drugs Dermatol. 2012;11(11):1342-6.

28. Han W, Ming M, He YY. Caffeine promotes ultraviolet B-induced apoptosis in human keratinocytes without complete DNA repair. J Biol Chem. 2011;286(26):22825-32.
29. Morphett L, Heath G, McIntosh W, Dorrian J. A Case Study Investigating a Behavioural Intervention to Reduce Caffeine Consumption. J Women Health Care. 2014;3:5. doi:10.4172/2167-0420.1000186

30. Alves RC, Oliveira SCeB. Benefícios do café na saúde: mito ou realidade? Quim Nova. 2009;32(8):2169-80. doi:10.1590/S0100-40422009000800031

31. Jee SH, He J, Whelton PK, Suh I, Klag MJ. The effect of chronic coffee drinking on blood pressure: a metaanalysis of controlled clinical trials. Hypertension. 1999;33(2):647-52. doi:10.1161/01.hyp.33.2.647

doi:10.1074/jbc.m111.222349 